FERMILAB-Pub-87/112-A

July 1987

\title{
QUARK MATTER IN THE HARTREE-FOCK APPROXIMATION
}

\author{
Frédérique Grassi") \\ NASA / Fermilab Astrophysics Center \\ Fermi National Accelerator Laboratory \\ Box 500 \\ Batavia, IL 60510-0500, U.S.A.
}

\begin{abstract}
A B S T R A T
An equation of state is computed for quark matter interacting through a phenomenological potential in the Hartree-Fock approximation. It is shown that for colour-independent confining potentials, it can be approximated by the Hartree result and leads to a first order mass phase transition. For colour-dependent confining potentials, a phase transition from a Fermi sphere to a Fermi shell is possible.
\end{abstract}

-) On leave from D.A.R.C.,Observatoire de Paris, 92195 Meudon, France 


\section{INTRODUCTION}

In order to discuss properties of the primordial quark-hadron phase transition or of condensed stellar objects, it is necessary to know the equation of state of the quark-gluon plasma. At sufficiently high densities or temperatures, when asymptotic freedom holds, perturbation-based equations of state may be used ${ }^{1}$. At low densities or temperatures, however, since we do not know how confinement occurs in QCD, one must resort to using phenomenological quark matter equations of state. Various such kinds of phenomenological equations of state have been proposed, each differing in the way confinement has been imposed: through boundary conditions as in the M.I.T. bag equation of state $^{2}$, through the acquisition of a large mass by quarks (coupled to a scalar field) outside hadrons as in soliton-bags ${ }^{3}$, etc.

In a previous article ${ }^{4}$, in the line of a paper by Olive $^{5}$, we derived, in the Hartree approximation, a phenomenological equation of state for quarks interacting through an interquark potential assumed to have a (positive) colour-independent infinitely rising - thus confining - component. It was shown that, first, if the confining potential was screened through pair creation ${ }^{6}$, the value of the thermodynamical quantities would be independent of the actual shape of the interquark potential. As a consequence of this, the equation of state depends on just one parameter, the constituent quark mass. Second it was seen that the quark plasma undergoes a first order phase transition, from a state of massive particles in a collective bound state at low densities, to a state of massless particles with a perfect gas behaviour at high densities - this being in agreement with what one would expect from QCD. Hence, while in the quark-hadron phase transition, one usually uses a different equation of state to describe each of the two phases, here a single equation of state is sufficient for the consideration of the whole transition.

However, in the Hartree approximation, the fermionic - anticommutating - character of 
quark operators is not taken into account. In order to do that, one has to include the Fock terms. These terms, as we show below, do depend on the shape of the interquark potential. It has be argued by $\mathrm{Chin}^{7}$ in the context of nuclear matter, that, at high density, the Hartree energy grows like $\mathbf{k}_{F}^{6}$ while the exchange - or Fock - energy behaves like $k_{F}^{4}$ so that the Fock corrections to the equation of state are expected to be small, at least at high densities. This was indeed checked, for all densities, by $\mathrm{Chin}^{7}$ and Horowitz \& Serot ${ }^{8}$. In Chin's argument, the $\mathrm{k}_{F}^{6}$ dominant term in the Hartree energy comes from a vector term. So this argument cannot be applied for instance to interquark potentials whose confining component is purely of a scalar type. (The one gluon exchange potential is vectorial but does not contribute in the Hartree approximation because of its colour dependence ${ }^{9,4}$ ). Hence the aim of this paper is the study of the effect of the Fock corrections on the equation of state, in the case of colour- independent confining potentials, and determine whether the Hartree approximation of paper 4 can be used without the Fock terms. For colour-dependent confining potentials, it is shown that the quark plasma cannot be described in the simple framework of a Fermi sphere-model as in the case of nucleons exchanging scalar and vector particles ${ }^{8}$.

\section{RELATIVISTIC HARTREE-FOCK APPROXIMATION}

For reasons already mentioned in reference 4 , the interquark potential will be assumed to have the following Lorentz structure

$$
V(r)=V_{V}(r) \gamma_{\mu}^{(1)} \gamma^{\mu(2)}-V_{S}(r) 1^{(1)} 1^{(2)}
$$

where

$V_{\mathrm{a}}(r)=\alpha V_{C}(r) 1^{(1)} 1^{(2)}$

$V_{v}(r)=\alpha V_{G}(r) \lambda^{(1)} \lambda^{(2)}-\beta V_{C}(r) \nu^{(1)} \nu^{(2)}$

$V_{G}(r)$ is the one gluon-exchange potential, $V_{C}(r)$ is a confining potential, 
$\nu$ is the colour unit matrix for colour-independant confining potential, and is a Gell-Mann matrix for colour dependant-confining potential, and either

(I) : $\alpha=1, \beta=0$, for pure scalar confining potentials,

or

(II): $\alpha, \beta \neq 0$, for confining potentials with both scalar and vector components.

Upon insertion in the (Dirac) equation of motion

$$
(i \not \partial-m) G(x, y)=\delta^{4}(x-y)-i \int d^{3} z G\left(x, z ; y, z^{+}\right) V(|x-z|)_{\mid z_{0}=x_{0}}
$$

of the Hartree-Fock approximation

$$
G(x, y ; z, t)=G(x, z) G(y, t)-G(x, t) G(y, z)
$$

and after a Fourier transform, one gets an equation for the two-point Green function

$$
\left[p-m-U_{H}-U_{F}(p)\right] G(p)=1
$$

where for colour-independent confining potentials

$U_{H}=\int d^{3} z\left[-i G\left(z, z^{+}\right] \nu_{C}(|x-z|)-P 1\right.$ with $\nu_{C}(r)=-\alpha V_{C}(r) 1^{(1)} 1^{(2)}-\beta V_{C}(r) \gamma_{\mu}^{(1)} \gamma^{\mu(2)}$ while for colour-dependent potentials

$U_{H}=-P 1$

and for both types of potentials

$U_{F}(p)=-\int \frac{d^{4} p^{\prime}}{(2 x)^{d}} G^{<}(|\vec{p}|) \tilde{V}\left(\left|\vec{p}-\vec{p}^{\prime}\right|\right)+P 1$

(in order to sum on all indices in $U_{H}$, one has to add and subtract a term P1; the same problem arises with $U_{F}$, but P1 appears with opposite $\operatorname{sign}^{9,4}$ ).

A glance at (2.1) and (2.4) suggests that $U_{H}$ and $U_{F}(|\vec{p}|)$ have only scalar and vector components. The coefficient of the vector component must be formed with the available four-vectors, i.e. the four-velocity, and for $U_{F}, p^{\mu}$. Hence in the rest frame of the plasma, $U_{H}$ and $U_{F}(|\vec{p}|)$ may be decomposed as

$$
U_{H}=U_{S}^{H} 1+U_{0}^{H} \gamma_{0}
$$




$$
U_{F}(|\vec{p}|)=U_{S}^{F}(|\vec{p}|) 1+U_{0}^{F}(|\vec{p}|) \gamma_{0}+U_{V}^{F}(|\vec{p}|) \vec{\gamma} \hat{p}
$$

Equation (2.4) may then be rewritten as

$$
\left[\gamma_{0} p_{H F}^{0}-\vec{\gamma} \vec{p}_{H F}-m_{H F}\right] G(p)=1
$$

where

$$
\begin{aligned}
p_{H F}^{0} & \equiv p_{0}-U_{0}^{H}-U_{0}^{F}(|\vec{p}|) \\
\vec{p}_{H F} & \equiv \vec{p}+\hat{p} U_{V}^{F}(|\vec{p}|) \\
m_{H F} & \equiv m+U_{S}^{H}+U_{S}^{F}(|\vec{p}|)
\end{aligned}
$$

This yields the following formula for the retarded propagator

$$
G^{<}(p)=\frac{1}{e^{\beta\left(p_{0}-\mu\right)}+1} A(p)
$$

where

$$
A(p)=\frac{\gamma_{0} p_{0}^{H F}-\vec{\gamma} \vec{p}_{H F}+m_{H F}}{2 \sqrt{\vec{p}_{H F}^{2}+m_{H F}^{2}}}\left[2 \pi \delta\left(p_{H F}^{0}-\sqrt{\vec{p}_{H F}^{2}+m_{H F}^{2}}\right)-2 \pi \delta\left(p_{H F}^{0}+\sqrt{\vec{p}_{H F}^{2}+m_{H F}^{2}}\right)\right]
$$

In order to compute the Hartree-Fock field defined in equation (2.4), only the matter part of $G^{<}(p)$ will be used. Because our model is phenomenological, vacuum fluctuations - which would otherwise give rise to infinities - should not show up. It is then straightforward to derive

$$
\begin{aligned}
U_{S}^{H}= & \left\{\int d^{3} r\left[-\alpha V_{C}(r)\right]\right\} \\
& 6 \int \frac{d^{3} p}{(2 \pi)^{3}} \frac{m_{H F}}{\sqrt{\vec{p}_{H F}^{2}+m_{H F}^{2}}}\left\{\frac{1}{\exp \left[\beta\left(U_{H F}^{0}+\sqrt{\vec{p}_{H F}^{2}+m_{H F}^{2}}-\mu\right)\right]+1}+\frac{1}{\exp \left[\beta \left(-U_{H F}^{0}+\sqrt{\left.\left.\vec{p}_{H F}^{2}+m_{H F}^{2}+\mu\right)\right]+1}\right.\right.}\right\} \\
U_{0}^{H}= & \left\{\int d^{3} r\left[-\beta V_{C}(r)\right]\right\} \\
& 6 \int \frac{d^{3} p}{(2 \pi)^{3}}\left\{\frac{1}{\exp \left[\beta\left(U_{H F}^{0}+\sqrt{\bar{p}_{H F}^{2}+m_{H F}^{2}}-\mu\right)\right]+1}-\frac{1}{\exp \left[\beta\left(-U_{H F}^{0}+\sqrt{\vec{p}_{H F}^{2}+m_{H F}^{2}}+\mu\right)\right]+1}\right\}
\end{aligned}
$$




$$
\begin{aligned}
& U_{S}^{F}=-\int \frac{d^{3} p}{(2 \pi)^{3}} \frac{m_{H F}}{2 \sqrt{\vec{p}_{H F}^{2}+m_{H F}^{2}}}\left\{-\alpha \tilde{V}_{C}(|\vec{p}-\vec{q}|)+4\left[\tilde{V}_{G}(|\vec{p}-\vec{q}|) \phi-\beta \tilde{V}_{C}(|\vec{p}-\vec{q}|)\right]\right\} \\
& \left\{\frac{1}{\exp \left[\beta\left(U_{H F}^{0}+\sqrt{\tilde{P}_{H F}^{2}+m_{H F}^{2}}-\mu\right)\right]+1}+\frac{1}{\exp \left[\beta \left(-U_{H F}^{0}+\sqrt{\left.\left.\overline{p_{H F}^{2}+m_{H F}^{2}}+\mu\right)\right]+1}\right.\right.}\right\} \\
& U_{0}^{F}=-\int \frac{d^{3} p}{(2 \pi)^{3}} \frac{1}{2}\left\{-\alpha \tilde{V}_{C}(|\vec{p}-\vec{q}|)-2\left[\tilde{V}_{G}(|\vec{p}-\vec{q}|) \phi-\beta \tilde{V}_{C}(|\vec{p}-\vec{q}|)\right]\right\} \\
& \left\{\frac{1}{\exp \left[\beta\left(U_{H F}^{0}+\sqrt{\bar{p}_{H F}^{2}+m_{H F}^{2}}-\mu\right)\right]+1}-\frac{1}{\exp \left[\beta\left(-U_{H F}^{0}+\sqrt{\hat{p}_{H F}^{2}+m_{H F}^{2}}+\mu\right)\right]+1}\right\} \\
& U_{V}^{F}=-\int \frac{d^{3} p}{(2 \pi)^{3}} \frac{\hat{q} \vec{p}}{2 \sqrt{\tilde{p}_{H F}^{2}+m_{H F}^{2}}}\left\{-\alpha \tilde{V}_{C}(|\vec{p}-\vec{q}|)-2\left[\tilde{V}_{G}(|\vec{p}-\vec{q}|) \phi-\beta \tilde{V}_{C}(|\vec{p}-\vec{q}|)\right]\right\} \\
& \left\{\frac{1}{\exp \left[\beta\left(U_{H F}^{0}+\sqrt{\hat{P}_{H F}^{2}+m_{H F}^{2}}-\mu\right)\right]+1}+\frac{1}{\exp \left[\beta\left(-U_{H F}^{0}+\sqrt{\hat{P}_{H F}^{2}+m_{H F}^{2}}+\mu\right)\right]+1}\right\}
\end{aligned}
$$

where $\phi=1$ for colour-dependent potential, and $\phi=16 / 3$ for colour-independent potential. (In what follows, we will concentrate for simplicity on case (I) of equation (2.1) $(\alpha=1, \beta=0)$. Note however that it was shown in reference 4 that in the Hartree approximation, case (II) is similar to case (I): only a mass transition, and no energy transition, is allowed and that, as far as the Fock terms are considered, the only difference between case (I) and case (II) is the factor in front of $\tilde{V}_{C}$.)

\section{INFRARED DIVERGENCES}

As in the Hartree approximation, the (infinitely rising) confining potential gives rise to infrared divergences. But because of the convolutions, the potential terms in the Fock components (2.8c-e) cannot be factorised as in the Hartree components (2.8a-b). The fact that the potential terms $\int d^{3} r\left[-\alpha V_{C}(r)\right]$ and $\int d^{3} r\left[-\beta V_{C}(r)\right]$ in the Hartree approximation could be factorised, had been used in reference 4 to compute their (finite) physical value and avoid having to specify the actual shape of the potential. In the Fock approximation, this trick cannot be applied: both the shape of the potential and the explicit way to remove 
infinities have to be specified. As for the shape of the potential, we will assume for simplicity

$$
V_{G}(r)=-a / r \text { and } V_{C}(r)=-(k r-U)
$$

Note that because of the minus sign in expression (2.1) for $V(r)$, the confining term is indeed repulsive at long distance (the eventual Gell-Mann matrices contribute with a plus sign in this approximation). The linear behaviour of the confining potential is strongly suggested by preliminary results in lattice $\mathrm{QCD}^{10}$, by string models ${ }^{11}$ and by explicit calculations in QCD with one time and one space dimension ${ }^{12}$. A large number of interquark potentials have also been proposed in quarkonium potential models (for recent reviews, see e.g. Bykov,Dremin \& Leonidov or Diekmann ${ }^{13}$ ), but they are usually mere interpolations between the onegluon exchange regime and the linear regime, so it is expected that our choice (3.1) will embody the gross features of the interquark interaction. To deal with the infinities, as in the Hartree approximation, one can argue that the Hartree- Fock components (2.8a-e) should not reach infinite values as pair creation between quarks at large enough distances takes place. In other words, physically one expects that the confining potential must be screened and not infrared divergent. In practice, we will assume that the interquark potential is exponentially smoothed, i.e. the scalar and vector components of $V_{C}$ will respectively be multiplied by $e^{-e_{1} r}$ and $e^{-c_{2} r}$. This procedure was followed for instance in reference 14 .

Ideally one would like to know the density dependence of $\mathrm{k}, \mathrm{U}, \mathrm{a}, c_{1}$ and $c_{2}$ to implement and solve (2.8a-e) but we have no way to compute it. However, it seems reasonable to think that since our purpose is to study the effect of the Fock corrections to the Hartree approximation, we will get, for densities of order some times the normal nuclear matter density, a qualitative understanding by keeping $\mathrm{k}, \mathrm{U}, \mathrm{a}, c_{1}$ and $c_{2}$ constant. In what follows, we have considered several sets of values for these parameters listed in table 1.

In addition, we will assume, for simplicity, that the screening lengths of the scalar confining potential and the (possible) vector confining potential are of the same order of 
magnitude and we will take

$$
c_{1}=c_{2}=300 . M e v \approx 1 / \text { radius of a light quarkonium. }
$$

(It is possible that the screening lengths be somehow larger than the radius of a light quarkonium, but the values of $\mathrm{k}, \mathrm{U}, \mathrm{a}$ and $m_{\mathrm{u}}$ given by potential models like the ones quoted in table 1, are computed for this range of distances. So we use it as well to be coherent).

\section{RESULTS}

The spectrum of energy (at $\mathrm{T}=0$ )

$$
E_{k}=U_{0}^{H}+U_{0}^{F}(k)+\sqrt{\vec{k}_{H F}(k)^{2}+m_{H F}(k)^{2}}
$$

is shown for colour-independent confining potentials in figure (1a) and for colour-dependent confining potentials in figure (1b), for the set of values given in Ono - the curves would be similar for other sets of values. It can be seen that the spectrum becomes inverted i.e. $E_{k=0}$ becomes greater than $E_{0<k<k_{f}}$ (where $k_{f}$ is the Fermi energy) at high densities in the case of colour-dependent confining potentials. This inversion cannot occur in the Hartree approximation alone: in this approximation, $U_{H}^{s}$ and $U_{H}^{0}$ do not depend on $\mathrm{k}$ (and $U_{H}^{v}$ is

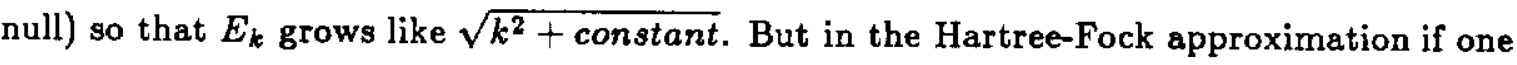
of the functions of $\mathrm{k}$ entering in (4.1) decreases, $E_{k}$ may be inverted as in figure (1b). Such a situation has also been encountered previously for nuclear matter in the Hartree-Fock approximation by Horowitz \& Serot ${ }^{16}$. Systems with inverted spectrum are unstable (a redistribution of levels can result in a state with lower energy) so we postpone their study and will concentrate in what follows on the study of colour-independent confining potentials.

In figure (2), the effective mass $m_{H F}$ has been plotted as a function of the chemical potential

$$
\mu=\sqrt{\vec{k}_{H F}\left(k_{f}\right)^{2}+m_{H F}\left(k_{f}\right)^{2}}+U_{0}^{H}+U_{0}^{F}\left(k_{f}\right)
$$


Two sets of values have been used: Ono and Ono with a somehow larger value of $U, U=1200$. Mev. For the first set, it can be noted that even if a mass phase transition is not possible in the Hartree approximation, it might occur in the Hartree-Fock approximation since in this case the HF-curve lies below the H-curve. The second set was chosen because it allows for a mass phase transition both in the Hartree and the Hartree-Fock approximation and is therefore more similar to the situation of reference 4 .

Once equations (2.8a-e) are solved -in other words once $m_{H F}, p_{H F}$ and $U_{H F}^{0}$ have been evaluated- the equation of state can be computed via

$$
\begin{aligned}
& \epsilon=3 \int \frac{d^{3} p}{(2 x)^{3}}\left\{U_{H F}^{0}\left\{\frac{1}{\exp \left[\beta\left(U_{H F}^{0}+\sqrt{\bar{p}_{H F}^{2}+m_{H F}^{2}}-\mu\right)\right]+1}-\frac{1}{\exp \left[\beta\left(-U_{H F}^{0}+\sqrt{\bar{p}_{H F}^{2}+m_{H F}^{2}}+\mu\right)\right]+1}\right\}\right. \\
& \left.+\left[\sqrt{\vec{p}_{H F}^{2}+m_{H F}^{2}}+\frac{\vec{p}_{H F} \vec{p}+m_{H F} m}{\sqrt{\vec{p}_{H F}^{2}+m_{H F}^{2}}}\right]\left\{\frac{1}{\exp \left[\beta \left(U_{H F}^{0}+\sqrt{\left.\left.\vec{p}_{H F}^{2}+m_{H F}^{2}-\mu\right)\right]+1}\right.\right.}+\frac{1}{\exp \left[\beta\left(-U_{H F}^{0}+\sqrt{\vec{p}_{H F}^{2}+m_{H F}^{2}}+\mu\right)\right]+1}\right\}\right\} \\
& p=3 \int \frac{d^{3} p}{(2 \pi)^{3}}\left\{U_{H F}^{0}\left\{\frac{1}{\exp \left[\beta \left(U_{H F}^{0}+\sqrt{\left.\left.\bar{p}_{H F}^{2}+m_{H F}^{2}-\mu\right)\right]+1}\right.\right.}-\frac{1}{\exp \left[\beta\left(-U_{H F}^{0}+\sqrt{\bar{p}_{H F}^{2}+m_{H F}^{2}}+\mu\right)\right]+1}\right\}\right. \\
& \left.+\left[\sqrt{\tilde{p}_{H F}^{2}+m_{H F}^{2}}+\frac{-\vec{p}_{H F} \vec{p} / 3-m_{H F} m}{\sqrt{\vec{p}_{H F}^{2}+m_{H F}^{2}}}\right]\left\{\frac{1}{\exp \left[\beta \left(U_{H F}^{0}+\sqrt{\left.\left.\vec{p}_{H F}^{2}+m_{H F}^{2}-\mu\right)\right]+1}\right.\right.}+\frac{1}{\exp \left[\beta\left(-U_{H F}^{0}+\sqrt{\bar{p}_{H F}^{2}+m_{H F}^{2}}+\mu\right)\right]+1}\right\}\right\}
\end{aligned}
$$

(these equations can be derived in the same way as for the Hartree approximation ${ }^{4}$ ).

In figure (3), the equation of state $p(\epsilon)$ has been plotted for the same two sets of parameters as in figure (2). While at low densities, the H-curves lie reasonably close to the HF-curves, at high densities, they lie further and further apart. This situation is different from that of the nuclear matter investigated by Horowitz \& Serot ${ }^{8}$. As mentionned in the introduction, this discrepancy can be easely understood: for nuclear matter at high density, the Hartree energy, which grows like $k_{F}^{6}$, dominates the Fock term which increases like $k_{F}^{4}$, but the Hartree energy of the quark matter studied in reference 4, does not have such a $k_{F}$ behaviour. However since we do not know the density dependence of the parameters $\mathrm{k}$, $\mathrm{U}, \mathrm{a}, c_{1}$ and $c_{2}$, the far end -or high density part- of these curves should not be taken into account anyway. Finally it is interesting to note that the effect of the Fock terms seems to 
be that the equation of state will reach its perfect gas stage $-p=\epsilon / 3-$ more quickly than in the pure Hartree case.

\section{CONCLUSION}

In this paper, we studied the effect of the Fock terms on the (Hartree) equation of state computed in reference 4. To achieve this, contrary to the Hartree case, we needed to specify the actual shape of the potential but we do not know the density dependence of the potential parameters. At low densities, where the calculations with fixed constant parameters can be considered as a reasonable approximation, it was seen that the Hartree first order phase transition from a gas of massive particles (in a collective bound state) to a state of massless particles survives when Fock terms are included and that the equation

of state is not very different. At moderate densities, the effect of the Fock terms seems to be that the quark plasma will reach its perfect gas behaviour $(p=\epsilon / 3)$ more rapidly.

Quark matter in the Hartree-Fock approximation has been studied previously by $\mathrm{E}$. Alvarez $^{17}$, who started from the usual QCD Lagrangian, and computed an equation of state. This equation of state always lies close to $p=\epsilon / 3$. Here confinement was added by hand and this led to a more reasonable behaviour of the quark matter at low density, in the Hartree-Fock approximation.

Phenomenological equations of state are usually needed for low densities where perturbative equations of state cannot be used, but it is interesting to investigate their high density limit. Here, the high density regime with both Hartree and Fock terms, given our lack of knowledge of the density dependance of the interquark parameters, is not reachable. However, one expects that, at these densities, the quark plasma should behave like a perfect gas, and this is actually what the Hartree equation of state $\mathrm{e}^{4}$ predicts. In this context, it may then be interesting to mention another paper by E. Alvarez ${ }^{18}$, who showed that for 
a large class of physical systems, the dominant term in the high density expansion of the partition function computed in the Hartree-Fock approximation, is the Hartree term.

\section{ACKNOWLEDGMENTS}

The author wishes to thank R. Hakim for useful discussions and B. Pichon for his help in solving sorne software problems. This work was supported in part by the DOE and by the NASA at Fermilab. 
FIGURE AND TABLE CAPTIONS

Figure 1: (a) Single particle energy spectrum $E_{k}$ as a function of momentum for quark matter intacting via a colour-independent confining potential with parameters as in Ono ${ }^{17}$.

(b) Same as in (a) but for colour-dependent confining potentials. Note the inversion of the spectrum.

Figure 2: Effective mass as a function of the chemical potential. The solid curve corresponds to a Hartree-Fock calculation with parameters as in Ono but $U=1200$. Mev and the dotted curve corresponds to a Hartree calculation with the same set of parameters. The dashed curve corresponds to a Hartree calculation with parameters as in Ono and the dash-dotted curve to a Hartree calculation with the same set of parameters.

Figure 3: Pressure as a function of energy density. See figure (2) for curve designation.

Table 1: Values of $k, U, a, c_{1}, c_{2}$ used in the interquark potential. 


\section{REFERENCES}

[1] For a panorama, see

J.C. Collins and M.J. Perry - Phys. Rev. Lett. 34 (1975) 1353;

G. Baym and S.A. Chin - Nucl. Phys. A262 (1976) 527;

S.A. Chin - Phys. Lett. 78B (1978) 552;

B.A. Freedman and L.D. Mc Lerran - Phys. Rev. D16 (1977) 1130;

ibid. D16 (1977) 1147; ibid. D16 (1977) 1169.

V. Baluni - Phys. Rev. D17 (1978) 2092;

E.V. Shuryak - Soviet Phys. JETP 47 (1978) 212;

J.I. Kapusta - Nucl. Phys. B148 (1978) 461;

T. Toimela - Phys. Lett. 142B (1983) 407; Int.J.Th.Phys. 24 (1985) 901.

[2] The original papers (based on ideas developped by Bogolioubov), are

A. Chodos, R.L. Jaffe, K. Johnson, C.B. Thorn and V.F. Weisskopf

- Phys. Rev. D12 (1974) 3471;

T.DeGrand, R.L. Jaffe, K. Johnson and J. Kiskis - Phys. Rev. D12 (1975) 2060.

[3] See for example

P. Vinciarelli - Lett. Nu. Cim. 4 (1972) 905;

W.A. Bardeen, M.S. Chanowitz, S.D. Drell, M. Weinstein and T.M. Yan

- Phys. Rev. D11 (1975) 1094;

R. Friedberg and T.D. Lee - Phys. Rev. D15 (1977) 1694;ibid. D16 (1977) 1096.

[4] F. Grassi - CERN preprint TH.4622/86.

[5] K.A. Olive - Nucl. Phys. B190 (1981) 483.

[6] J. Kogut and L. Susskind - Phys. Rev. Lett. 34 (1975) 767.

[7] S.A. Chin - Ann. Phys. 108 (1977) 301.

[8] C.J. Horowitz and B.D. Serot - Phys. Lett. 109B (1982) 341; 
- ibid. 108B (1982) 377; - Nucl. Phys. A399 (1983) 528.

[9] C. Kittel - "Quantum Theory of Solids" (Wiley, 1963).

[10] M. Creutz - "Quarks, Gluons \& Lattices" (Cambridge, 1983).

[11] J. Kogut and G. Parisi - Phys. Rev. Lett. 47 (1981) 1089.

[12] G. t'Hooft - Nucl. Phys. B75 (1974) 461.

[13] A.A. Bykov, I.M. Dremin and A.V. Leonidov - Sov. Phys. Usp. 27 (1984) 234

B. Diekmann - preprint Bonn-HE-84.

[14] A.B. Henriques, B.H. Kellett and R.G. Moorhouse, Phys. Lett. 64B (1976) 85.

[15] S.Godfrey and N.Isgur - Phys. Rev. D32 (1985) 189.

M.Arafah, R.Bhandari and B.Ram - Lett. Nuovo Cimento 38 (1983) 305.

S.Ono - Phys. Rev. D26 (1982) 2510.

D.P.Stanley and D.Robson - Phys. Rev. Lett. 45 (1980) 235.

[16] C.J.Horowitz and B.D.Serot - Phys. Lett. 109B (1982) 341;

ibid. 108B (1982) 377; Nucl.Phys.A399 (1983) 529.

[17] E.Alvarez - Phys. Rev. D23 (1981) 1715.

[18] E.Alvarez - Phys. Lett. 110B (1982) 315. 
Table 1

\begin{tabular}{|l|l|l|}
\hline Authors [15] & Values & Methods \\
\hline Godfrey & $k=0.13510^{6} \mathrm{Mev}^{2}$ & Klein-Gordon for all the mesons and \\
\& Isgur & $a(1000 \mathrm{Mev})=0.6$ & quarkonia \\
(1985) & $U=190 . \mathrm{Mev}$ & \\
& $m_{u}=220 . \mathrm{Mev}$ & \\
Arafah, & $k=0.110^{6} \mathrm{Mev}^{2}$ & Bethe-Salpeter for quarkonia \\
Bhandari \& & $a=0.8$ & \\
Ram (1983) & $U=150 . \mathrm{Mev}$ & \\
& $m_{u}=300 . \mathrm{Mev}$ & \\
Ono & $k=0.15610^{6} \mathrm{Mev}^{2}$ & Klein-Gordon for quarkonia \\
(1982) & $a(1000 \mathrm{Mev})=0.74$ & \\
& $U=640 . \mathrm{Mev}$ & \\
Bradley & $m_{u}=290 . \mathrm{Mev}$ & \\
\& Robson & $k=0.1610^{6} \mathrm{Mev}$ & Schrödinger for light mesons \\
(1980) & $a=0.16$ & \\
& $U=630 . \mathrm{Mev}$ & \\
& $m_{u}=340 . \mathrm{Mev}$ & \\
\hline
\end{tabular}




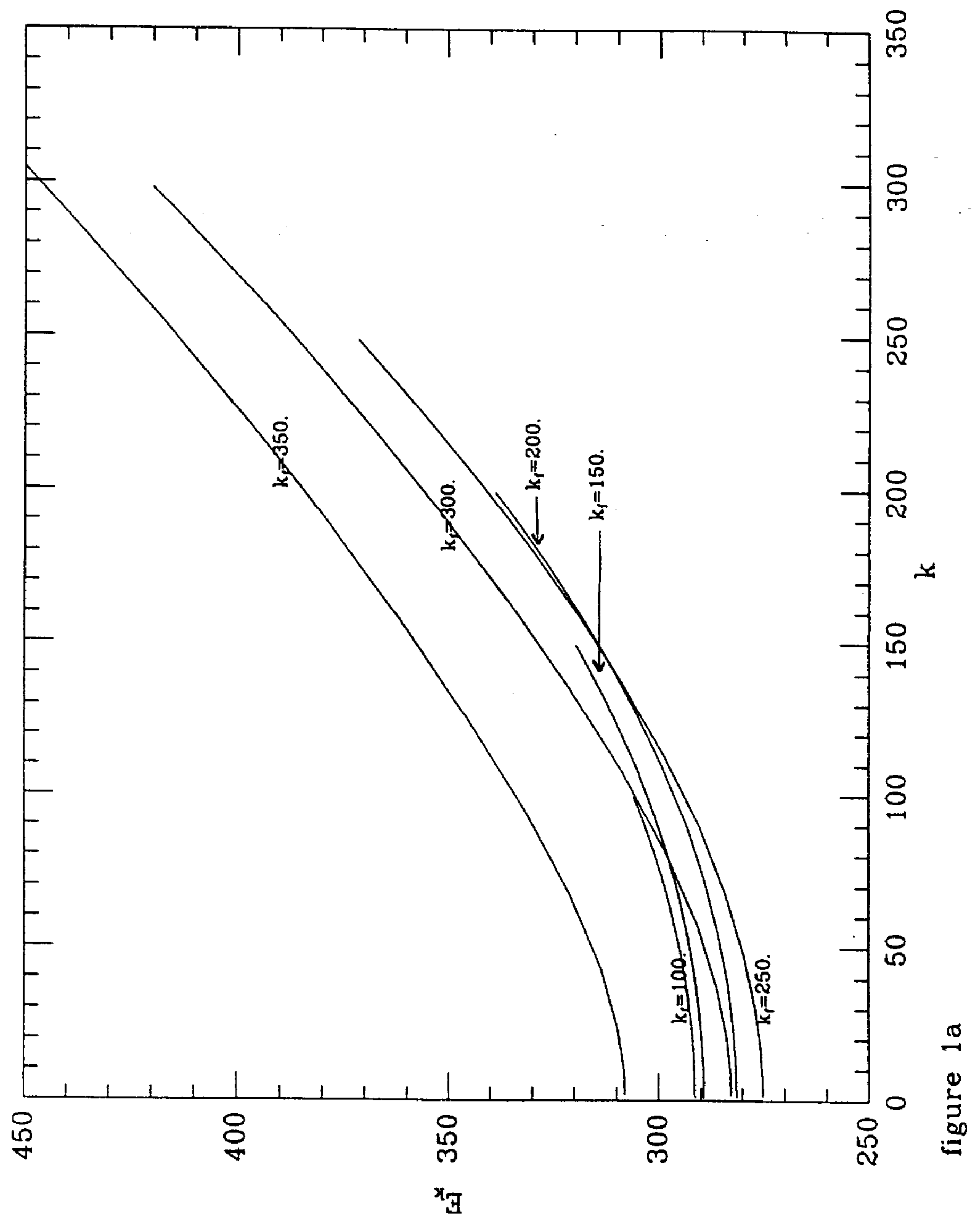




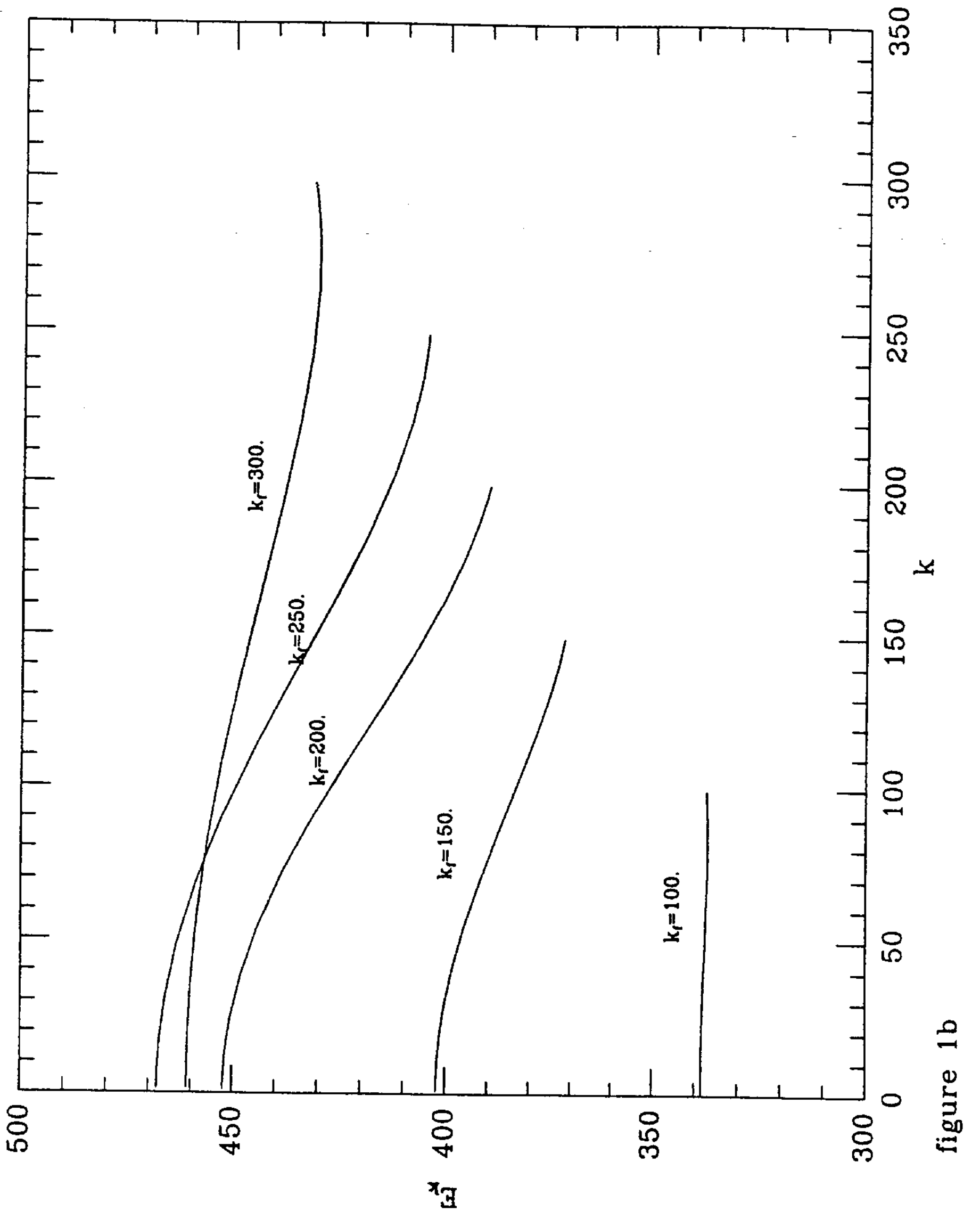

$-17-$ 


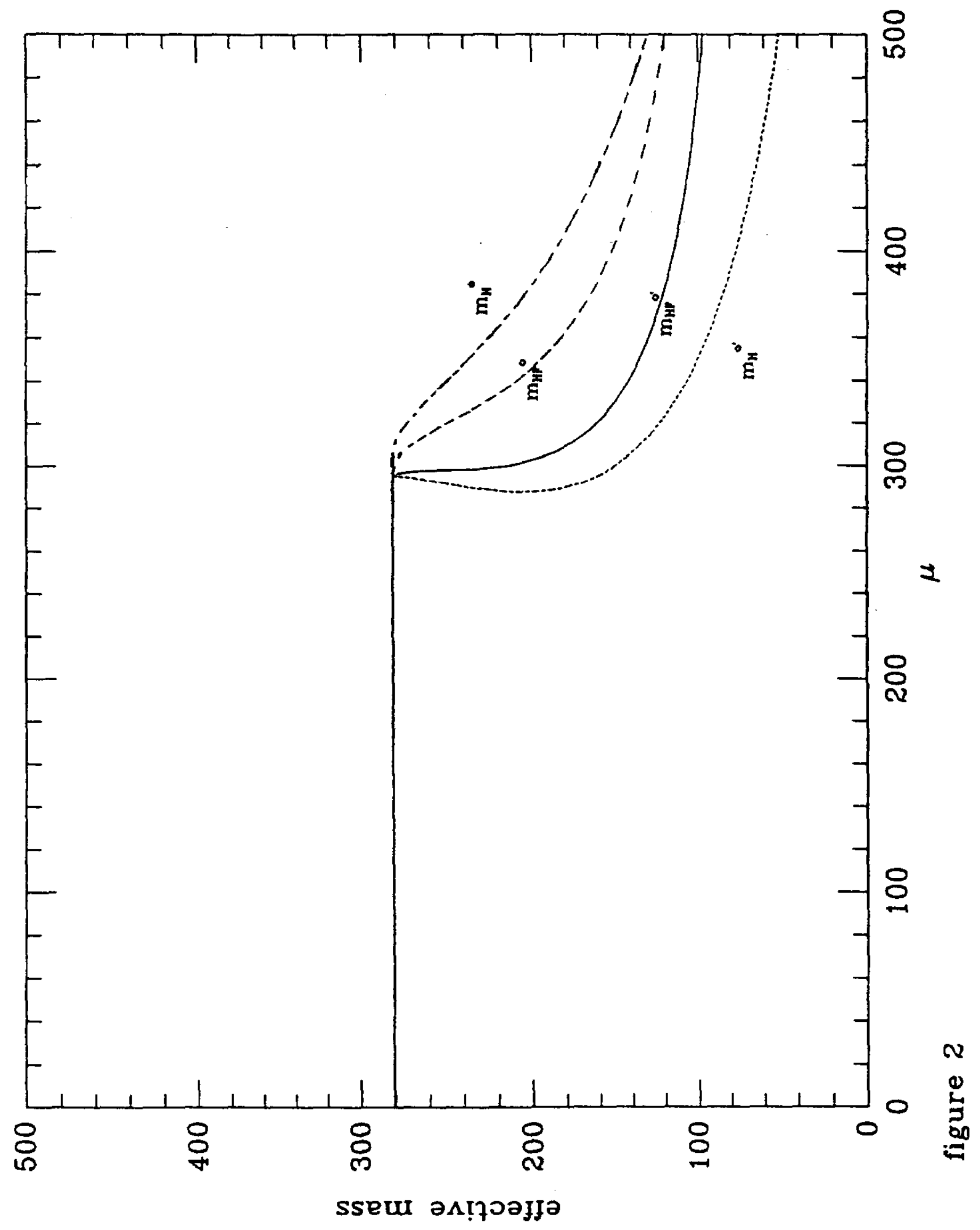




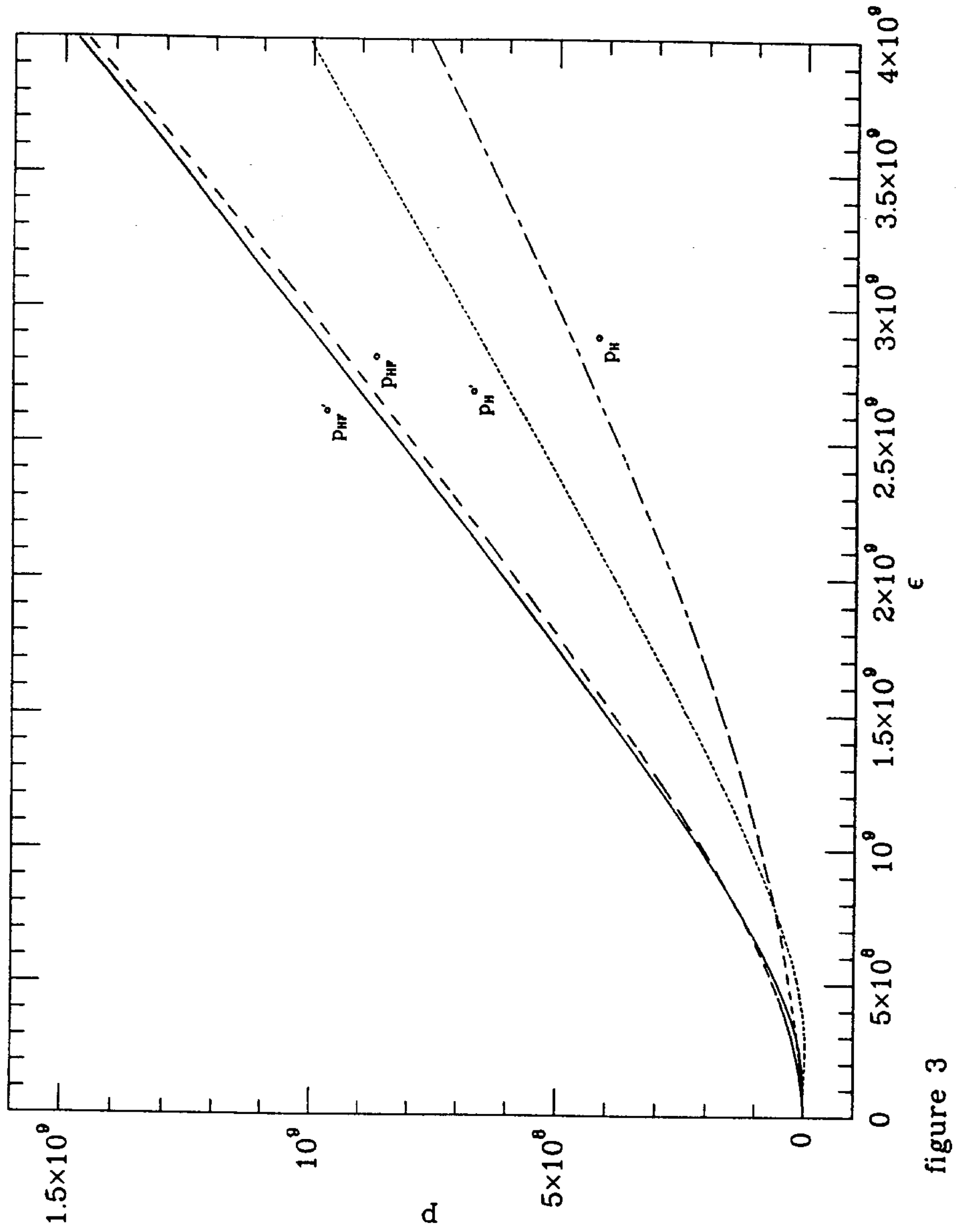

